\title{
Long-term renal function and hypertension in adult survivors of childhood sarcoma: Single center experience
}

Amalia Schiavetti, Valeria Pedetti, Giulia Varrasso, Oriana Marrucci, Camilla Celani, Gianmarco Andreoli \& Enea Bonci

To cite this article: Amalia Schiavetti, Valeria Pedetti, Giulia Varrasso, Oriana Marrucci, Camilla Celani, Gianmarco Andreoli \& Enea Bonci (2018) Long-term renal function and hypertension in adult survivors of childhood sarcoma: Single center experience, Pediatric Hematology and Oncology, 35:3, 167-176, DOI: 10.1080/08880018.2018.1476941

To link to this article: https://doi.org/10.1080/08880018.2018.1476941

舟 Published online: 19 Sep 2018.

Submit your article to this journal $\sqsubset$

Llll Article views: 31

View Crossmark data ¿ 


\title{
Long-term renal function and hypertension in adult survivors of childhood sarcoma: Single center experience
}

\author{
Amalia Schiavetti ${ }^{\mathrm{a}}$, Valeria Pedetti ${ }^{\mathrm{a}}$, Giulia Varrasso ${ }^{\mathrm{a}}$, Oriana Marrucci ${ }^{\mathrm{a}}$, \\ Camilla Celani ${ }^{\mathrm{a}}$, Gianmarco Andreoli ${ }^{\mathrm{b}}$, and Enea Bonci ${ }^{\mathrm{b}}$ \\ ${ }^{a}$ Department of Pediatrics, Sapienza University, Rome, Italy; ${ }^{b}$ Department of Experimental Medicine, \\ Sapienza University, Rome, Italy
}

\begin{abstract}
Aim: Little data is available on long-term renal impairment in survivors from childhood sarcoma. We investigated the prevalence of renal impairment and hypertension after very long-term follow-up in survivors who reached adulthood after treatment for childhood sarcoma. Methods: A cross-sectional single center study was performed. Outcomes included estimating glomerular filtration rate (eGFR), albuminuria, glycosuria, serum phosphate and magnesium, tubular reabsorption phosphate (TRP), chronic kidney disease (CKD) according to the "Kidney Disease: Improving Global Outcomes" (KDIGO) guidelines and blood pressure (BP). Results: Out of 87 $>5$ year sarcoma survivors, 30 adults $(10 \mathrm{~F} / 20 \mathrm{M}$, median age at diagnosis 9 years, median age at investigation 26 years, median follow-up 16 years, mean 19 years) were identified. Renal impairment was detected in four cases (13.3\%); three of these fulfilled the criteria for CKD. Among the adult survivors, a subgroup of 15 cases (50\%) had received ifosfamide without confounding factors such as a diagnosis of genito-urinary rhabdomyosarcoma or administration of other potentially nephrotoxic chemotherapy (platinum-based drugs or methotrexate); no renal dysfunction was detected in this subgroup. In the whole cohort of sarcoma survivors, hypertension was diagnosed in four cases (13.3\%); BP was significantly correlated with body mass index [ $p$.014]. Conclusion: In our series of adult survivors treated for a diagnosis of sarcoma in their childhood, the prevalence of CKD was $10 \%$. We found survivors treated with ifosfamide as the only nephrotoxic agent did not present glomerular or tubular toxicity at long term follow-up, but further studies including a larger number of cases are required to confirm it.
\end{abstract}

\section{ARTICLE HISTORY}

Received 27 September 2017

Revised 26 April 2018

Accepted 2 May 2018

\section{KEYWORDS}

Children; hypertension;

renal function;

sarcoma; survivors

\section{Introduction}

The increased survival rate for children with cancer has led to the recognition of chronic health conditions related to previous therapy in long term survivors. ${ }^{1-3}$ Late treatment sequelae may be responsible for chronic diseases and reduced life expectancy in survivors. Among the late effects of childhood cancer therapy are renal impairment and associated hypertension. Occasionally, malignant disease itself may cause renal 
impairment by damaging normal renal tissue by tumor infiltration or by urinary tract obstruction. More commonly, nephrotoxicity is a known side effect of certain childhood cancer therapies, resulting in the decline of glomerular filtration rate (GFR), the deterioration of tubular function, the development of albuminuria and hypertension. Potentially nephrotoxic agents include ifosfamide, the platinum compounds cisplatin and carboplatin, methotrexate, radiotherapy involving the kidney region. ${ }^{4-8} \mathrm{~A}$ recent Cochrane systematic review on nephrotoxic childhood cancer treatments reported a prevalence of long-term renal impairment ranging from 0 to $84 \%{ }^{9}$ This gap may be due to multiple factors. Data is not homogenous respect to the malignancies, treatments, outcomes measurements and time of follow-up. In particular, drug-renal adverse effects are common during therapy or at short-term follow-up, but little data is available on renal function impairment in adult survivors from childhood cancer and the evidence about the long-term renal function impairment remains inconclusive. As more and more patients reach adulthood, long-term effects of specific nephrotoxic agents have become the focus of research to better define high-risk cases and to better design effective surveillance strategies. Survivors with initial impaired renal function are usually symptom free, but even in subjects with subclinical toxicity, the potential for serious morbidity in later life is worrying. Chronic kidney disease (CKD) and hypertension are two important and treatable cardiovascular risk factors. Survivors from soft tissue sarcoma or from bone sarcoma may be at risk for nephrotoxicity because they are usually treated by one or more potentially nephrotoxic drugs or because the disease involves the genitourinary system.

In this study, we have invited the adult survivors of childhood sarcoma treated in a single centre to receive a specific screening with the aim to evaluate the prevalence of long term renal impairment and hypertension. To better evaluate the ifosfamide-related nephrotoxicity, we selected among these survivors those who received ifosfamide without confounding factors such as a diagnosis of genito-urinary rhabdomyosarcoma (RMS) or chemotherapy with platinum-based drugs or methotrexate.

\section{Patients and methods}

After institutional authorization a cross-sectional single-center study was performed. Demographic and clinical data were collected from the medical files of patients treated for sarcoma at the Pediatric Oncology Unit of "Sapienza" University of Rome. Eligibility criteria for the current study were as follows: diagnosis and treatment for sarcoma since 1980; age $\leq 21$ years at diagnosis; age at study $\geq 17$ years, survival $>5$ years after diagnosis, no high-dose chemotherapy with stem cell rescue, no kidney radiotherapy or nephrectomy, no secondary tumor development. Follow-up time was defined as time since cessation of treatment until renal function measurement. All eligible survivors were invited to the outpatient clinic to receive specific screening. Informed consent was obtained, as appropriate. Survivors underwent a physical examination, chemistry, kidney ultrasound, urine analysis, and blood pressure (BP) measurement. They were matched for body mass index (BMI) because of the potential influence of body composition on renal function and BP. 
Nephrotoxic agents were defined as follows: administration of ifosfamide, platinumbased drugs (cisplatin or carboplatin) or methotrexate and a diagnosis of genito-urinary RMS. To better evaluate the ifosfamide-related nephrotoxicity, we selected adult sarcoma survivors in two groups, those who received ifosfamide as the only nephrotoxic agent (group 1), and those who received multiple nephrotoxic agents (group 2).

\section{Chemistry}

For renal function evaluation, chemistry has included the determination of serum electrolytes, $\mathrm{Ca}, \mathrm{Mg}, \mathrm{P}, \mathrm{BUN} /$ creatinine and the determination of urine protein, creatinine, glucose, and phosphate. Serum triglycerides $>1.7 \mathrm{mmol} / \mathrm{L}$, high density lipoprotein cholesterol $<1.03 \mathrm{mmol} / \mathrm{L}$ for men, and $<1.29 \mathrm{mmol} / \mathrm{L}$ for women, and glucose $>7 \mathrm{mmol} / \mathrm{L}$ were considered for diagnosis of diabetes and/or metabolic disease.

\section{Renal function}

Outcome measurements has included estimating (e)GFR, albuminuria, serum phosphate/hypophosphatemia, serum magnesium/hypomagnesaemia, tubular reabsorption phosphate (TRP), kidney ultrasound and BP.

eGFR was calculated from serum creatinine standardized to isotope dilution mass spectrometry (IDMS), in a single laboratory to avoid interlaboratory error in creatinine determinations. ${ }^{10}$ Estimated glomerular function was calculated by the Chronic Kidney Disease Epidemiology Collaboration (CKD-EPI) equation. ${ }^{11}$ We defined decreased GFR as an estimated GFR $<90 \mathrm{~mL} / \mathrm{min} / 1.73 \mathrm{~m}^{2}$ and decline in GFR category ( $\geq 90$ [G1], 60-89 [G2], 45-59 [G3a], 30-44 [G3b], 15-29 [G4], <15 [G5] mL/min/1.73 m²), as recommended by "Kidney Disease: Improving Global Outcomes" (KDIGO) guidelines. ${ }^{12}$

Albuminuria was evaluated using a 24-h urine collection for albumin excretion; albumin urine concentrations were assessed using the immunological method. Albuminuria was expressed according to A1-3 categories by KDIGO guidelines. ${ }^{12} \mathrm{~A}$ threshold for urinary albumin excretion of $\geq 30 \mathrm{mg} / 24 \mathrm{~h}$ sustained for $>3$ months was considered pathological.

Tubular function was assessed by measuring serum phosphate, magnesium, and other electrolytes, by measuring glycosuria, urine $\mathrm{PH}$, and by the determination of tubular reabsorption phosphate (TRP). Serum phosphate, magnesium, and other electrolytes were considered normal if the value was in the normal range and replacement therapy was not being administered. TRP was calculated by the following formula: $\left(1-\frac{\mathrm{Cr} \mathrm{S} \times \mathrm{P} \mathrm{U}}{\mathrm{Cr} \text { X P S }}\right) \times 100(\mathrm{Cr}$, creatinine; P, phosphate; S, serum; U, urinary), it was considered in normal value $\geq 80 \%$.

Kidney ultrasound was assessed for signs of kidney damage (nephrocalcinosis, cysts, hydronephrosis, stones, and scars).

Persistent eGFR $<60 \mathrm{~mL} / \mathrm{min} / 1.73 \mathrm{~m}^{2}$ or albuminuria or tubular dysfunction or abnormalities in kidney structure were considered as CKD. An eGFR $<90>60 \mathrm{~mL} / \mathrm{min} /$ $1.73 \mathrm{~m}^{2}$ (G2 category) without other markers of kidney damage was not considered CKD. ${ }^{12}$ The subjects that presented with $\mathrm{CKD}$ at the initial risk assignment were reassessed after 3 or 6 months. 


\section{Blood pressure}

BP was measured in every case by the auscultatory method using a standard sphygmomanometer and a stethoscope and it was defined as hypertensive if systolic BP was $\geq 140 \mathrm{mmHg}$, diastolic BP was $\geq 90 \mathrm{mmHg}$, or any antihypertensive medication was used. BP was measured three times and the lowest BP determination was recorded. Pre-hypertension was not recorded. Possible correlation was investigated between BMI and systolic BP.

\section{Statistical analysis}

The comparison between frequency of eGFR $<90 \mathrm{~mL} / \mathrm{min} / 1.73 \mathrm{~m}^{2}$, of TRP, and of CKD was analyzed using two tails Fisher's Exact Test. Correlation between systolic BP and BMI was performed using Spearman's Test Rho. The results were considered statistically significant if the $p$ value was $<.05$. Time (year) referred to patient age and follow-up were rounded up and down.

\section{Results}

\section{Sarcoma survivors}

Out of 87 five-year survivors, 42 were adults who presented with the inclusions criteria for the study. Twelve of these were lost or refused to participate, thus 30 cases were investigated; their characteristics are shown in the Table 1 . Ten were females and 20 males, the median age at diagnosis was 9 years (range 0.8-21), mean 9 years, SD 6.27. Twenty-one had been treated for RMS, 2 for soft tissue sarcoma, 4 for Ewing sarcoma (ES), and 3 for osteosarcoma. The median follow-up was 16 years (range 6-34), mean 19 years, SD 7.79. The median age at investigation was 26 years (range 17-47 years), mean 28 years, SD 8.58. Patients lost to follow-up were similar to those evaluated in terms of sex $(5 \mathrm{~F}, 7 \mathrm{M})$, median age at treatment (10 years), malignancy (10 RMS, $1 \mathrm{ES}$, 2 soft tissue sarcoma). No patient received high-dose chemotherapy with stem cell rescue or whole abdomen radiotherapy or radiotherapy on kidney field. Twenty-four cases had received local radiotherapy, three of these on bladder site. Among 30 adult sarcoma survivors, 11 had $\mathrm{BMI} \geq 25$ and another one had BMI $\geq 36$. Two of these cases fulfilled the criteria for metabolic disease.

Group 1 consisted of 15 out of 30 adult sarcoma survivors (50\%). Group 1 (5 females and 10 males) had a median age at diagnosis of 13 years (range 2-21), a median age at investigation of 28 years (range 17-47), and a median follow-up of 17 years (range 7-28). Median ifosfamide cumulative dose was $54 \mathrm{~g} / \mathrm{m}^{2}$ (range $18-106$ ), mean $60.9 \mathrm{~g} / \mathrm{m}^{2}$, SD 24.7; ifosfamide was associated with vincristine, actinomycin D or doxorubicin or etoposide.

Group 2 sarcoma survivors (5 females and 10 males) had a median age at diagnosis of 6 years (range $0.8-17$ ), a median age of investigation of 23 years (range 17-46), and a median follow-up of 16 years (range 6-34). They received multiple nephrotoxic agents: ifosfamide (median cumulative dose of $48 \mathrm{~g} / \mathrm{m}^{2}$ ), other nephrotoxic drugs or surgery/ radiotherapy for bladder RMS. 
Table 1. Characteristics and renal function in sarcoma survivors.

\begin{tabular}{|c|c|}
\hline Variables & Survivors $(n 30)$ \\
\hline \multicolumn{2}{|c|}{ Age at diagnosis (yrs) } \\
\hline median (range) & $9(0.8-21)$ \\
\hline \multicolumn{2}{|l|}{ Age at study (yrs) } \\
\hline median (range) & $26(17-47)$ \\
\hline \multicolumn{2}{|l|}{ Follow-up (yrs) } \\
\hline median (range) & $16(6-34)$ \\
\hline \multicolumn{2}{|c|}{$\begin{array}{l}\text { Nephrotoxic chemotherapy } \\
n \text { (frequency) }\end{array}$} \\
\hline Only Ifo & $17(57 \%)$ \\
\hline Carbo & $7(23 \%)$ \\
\hline Mtx & $3(10 \%)$ \\
\hline Cis & $3(10 \%)$ \\
\hline \multicolumn{2}{|l|}{ Radiotherapy } \\
\hline \multicolumn{2}{|l|}{$n$ (frequency) } \\
\hline Kidney region & 0 \\
\hline Bladder & $3(10 \%)$ \\
\hline Other sites & $21(70 \%)$ \\
\hline Not & $6(20 \%)$ \\
\hline \multicolumn{2}{|c|}{ Albuminuria $>30 \mathrm{mg} / 24 \mathrm{~h}$} \\
\hline$n$ (frequency) & $2(6.6 \%)$ \\
\hline \multicolumn{2}{|c|}{$\mathrm{eGFR} \mathrm{mL/min/1.73} \mathrm{m}^{2}$} \\
\hline \multicolumn{2}{|c|}{$n$ (frequency) } \\
\hline$<90$ & $2(6.6 \%)$ \\
\hline$<60$ & $1(3.3 \%)$ \\
\hline \multicolumn{2}{|l|}{ TRP $<80 \%$} \\
\hline$n$ (frequency) & $2(6.6 \%)$ \\
\hline \multicolumn{2}{|l|}{ Glicosuria } \\
\hline$n$ & 0 \\
\hline \multicolumn{2}{|l|}{ hypoMg } \\
\hline$n$ (frequency) & 0 \\
\hline \multicolumn{2}{|l|}{ hypoP } \\
\hline$n$ (frequency) & $1(3.3 \%)$ \\
\hline \multicolumn{2}{|l|}{ CKD } \\
\hline$n$ (frequency) & $3(10 \%)$ \\
\hline
\end{tabular}

\section{Renal function}

The mean eGFR in all cohort of survivors was $110.6 \mathrm{~mL} / \mathrm{min} / 1.73 \mathrm{mq}$ (103.6-118.0 95\% C.I.).

Renal impairment was detected in four cases (13.3\%) Table 1.

Case $n$ 1: eGFR $<60 \mathrm{~mL} / \mathrm{min} / 1.73 \mathrm{~m}^{2}$, albuminuria A2 category, TRP $49 \%$, hypophosphatemia, urine $\mathrm{PH}>7$.

Case $n$ 2: eGFR $<90 \mathrm{~mL} / \mathrm{min} / 1.73 \mathrm{~m}^{2}$ and TRP $76 \%$.

Case $n$ 3: albuminuria A2 category

Case $n$ 4: eGFR $<90 \mathrm{~mL} / \mathrm{min} / 1.73 \mathrm{~m}^{2}$

Cases $n$ 1, 2, 3 fulfilled the criteria for CKD according to KDIGO guidelines. They were referred to a nephrology unit. Two of these $(n 1$ and $n 3)$ had received surgery (cystectomy/partial cystectomy) and radiotherapy for bladder RMS. In particular, case $n 1$ with CKD at stage $3 \mathrm{a}$ is continuing screening and replacement therapy in a nephrology center.

Serum magnesium, calcium and electrolytes were in the normal range with no replacement therapy in the remaining 29 cases. 
Table 2. Characteristics of survivors treated with ifosfamide without cisplatin, carboplatin, methotrexate and without diagnosis of bladder rhabdomyosarcoma.

\begin{tabular}{|c|c|c|c|c|c|c|c|c|c|c|c|}
\hline $\mathrm{Pt}$ & $\mathrm{H}$ & $\begin{array}{c}\text { AgeD } \\
\text { yrs }\end{array}$ & $\begin{array}{c}\text { Ages } \\
\text { yrs }\end{array}$ & $\begin{array}{l}\text { FU } \\
\text { yrs }\end{array}$ & $\begin{array}{c}\text { Total dose IFO } \\
\mathrm{g} / \mathrm{m}^{2}\end{array}$ & $\begin{array}{c}\mathrm{A} \\
\mathrm{mg} / 24 \mathrm{~h}\end{array}$ & eGFR & TRP & $\mathrm{Mg}$ & $P$ & CKD \\
\hline 1 & RMS & 2 & 18 & 15 & 54 & 12.9 & 135 & $86 \%$ & 1.9 & 3.7 & no \\
\hline 2 & ES & 17 & 28 & 11 & 106 & 2.2 & 118 & $97 \%$ & 2.2 & 3.4 & no \\
\hline 3 & ES & 14 & 33 & 19 & 27 & 4.1 & 122 & $86 \%$ & 2.1 & 2.8 & no \\
\hline 4 & RMS & 13 & 21 & 7 & 54 & 2 & 107 & $91 \%$ & 2.1 & 4.3 & no \\
\hline 5 & RMS & 21 & 47 & 24 & 54 & 2.9 & 112 & $81 \%$ & 1.9 & 3.4 & no \\
\hline 6 & RMS & 17 & 39 & 22 & 18 & 6.7 & 96 & $80 \%$ & 2.3 & 2.8 & no \\
\hline 7 & RMS & 20 & 28 & 9 & 54 & 2.4 & 102 & $88 \%$ & 2.1 & 3.4 & no \\
\hline 8 & RMS & 6 & 25 & 18 & 54 & 3.4 & 93 & $92 \%$ & 2 & 4 & no \\
\hline 9 & RMS & 9 & 29 & 18 & 54 & 9 & 115 & $81 \%$ & 2 & 3.4 & no \\
\hline 10 & RMS & 16 & 33 & 17 & 90 & 1 & 92 & $92 \%$ & 2.1 & 3.7 & no \\
\hline 11 & RMS & 6 & 33 & 27 & 54 & 1.3 & 99 & $86 \%$ & 2 & 4 & no \\
\hline 12 & ES & 17 & 28 & 11 & 102 & 12 & 129 & $90 \%$ & 2 & 3.4 & no \\
\hline 13 & RMS & 5 & 17 & 11 & 54 & 10 & 140 & $92 \%$ & 2.2 & 4.6 & no \\
\hline 14 & RMS & 2 & 29 & 28 & 84 & 3.4 & 133 & $90 \%$ & 1.8 & 3.7 & no \\
\hline 15 & RMS & 9 & 24 & 15 & 54 & 4.6 & 133 & $94 \%$ & 2.1 & 3.7 & no \\
\hline
\end{tabular}

Note. Pt: patient; H: histology; yrs: years; FU: follow-up; IFO: ifosfamide; A: albuminuria; eGFR: estimated glomerular filtration rate; TRP: tubular reabsorption phosphate; Mg: magnesium; P: phosphate; RMS: rhabdomyosarcoma; ES: Ewing sarcoma; ARMS: alveolar rhabdomyosarcoma; ERMS: embryonal rhabdomyosarcoma; BRMS: botryoides rhabdomyosarcoma; D: diagnosis; S: study.

Table 3. Renal function in group 1 sarcoma survivors (ifosfamide without other nephrotoxic agents) and group 2 sarcoma survivors.

\begin{tabular}{lccl}
\hline Variables & Group 1 & Group 2 & \\
\hline Mean eGFR & $n 15$ & $n 15$ & $p$ Value \\
eGFR $<90$ & $114.5 \pm 15.9$ & $105.73 \pm 22.6$ & n.s. \\
$n$ & 0 & 3 & $p=.05$ \\
CKD & 0 & 3 & $p=.05$ \\
$n$ & 0 & 2 & n.s. \\
TRP $<80 \%$ & 0 & 1 & n.s. \\
$n$ & 0 & &
\end{tabular}

Note. $n$ : number; IFO: ifosfamide; eGFR: estimated glomerular filtration rate; CKD: chronic kidney disease; TRP: tubular reabsorption phosphate; HypoP: hypophosphatemia.

Group 1 sarcoma survivors presented with a mean eGFR of $115 \mathrm{~mL} / \mathrm{min} / 1.73 \mathrm{~m}^{2}$ and had no G2 or G3 category or tubular dysfunction or CKD (Table 2). Group 1 sarcoma survivors had a better renal function in comparison with group 2 sarcoma survivors (Table 3).

\section{Hypertension}

Four (13.3\%) out of 30 sarcoma survivors had hypertension. Systolic BP was significantly correlated with BMI ( $p .014$; Figure 1).

\section{Discussion}

In this study, we investigated the long term renal function assessed by KDIGO criteria in adult survivors of childhood sarcoma after a median follow-up of 16 years, (mean 19 years; range 6-34 years). The prevalence of renal impairment was $13.3 \%$. Three (10\%) 


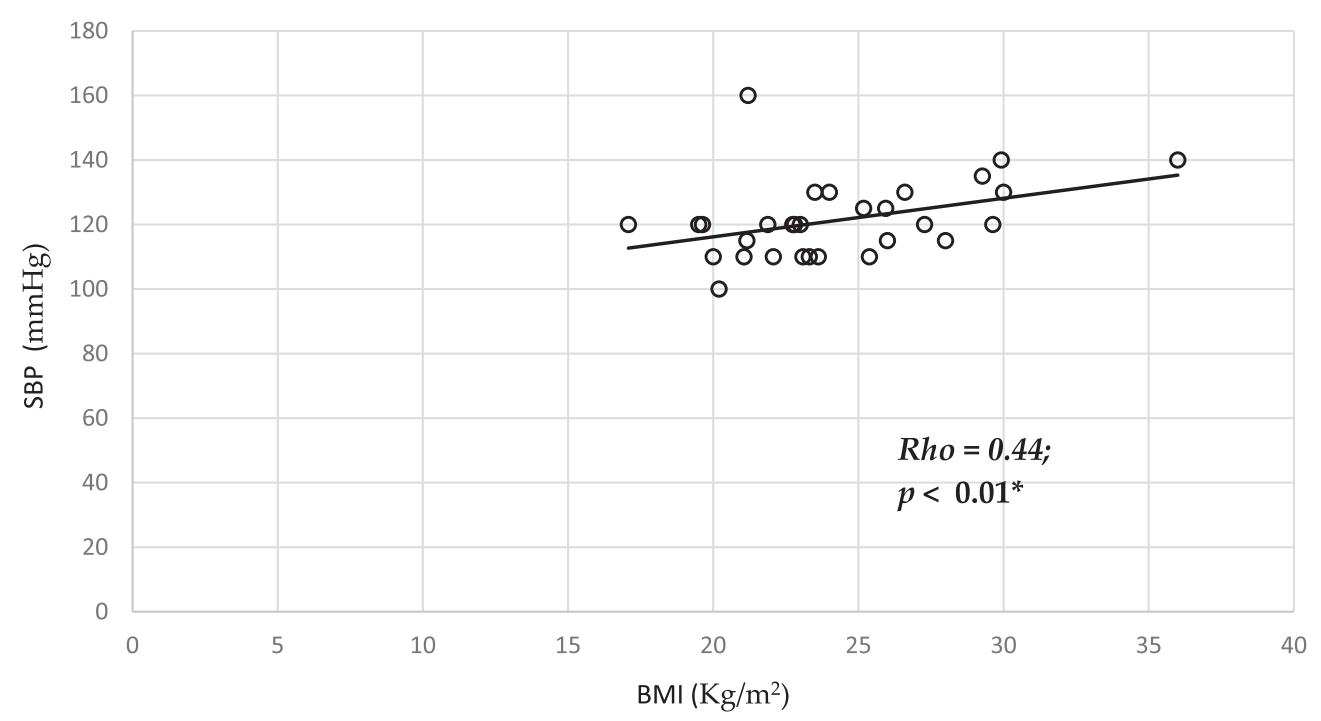

Figure 1. Systolic blood pressure as function of body mass index in sarcoma survivors. BMl: body mass index; SBP: systolic blood pressure.

survivors fulfilled the criteria for CKD, but two of them had received surgery (cystectomy/partial cystectomy) and radiotherapy for bladder rhabdomyosarcoma. Actually, $40 \%$ of bladder/prostate rhabdomyosarcoma survivors had decreased renal function, thus we considered this site of disease as a potentially nephrotoxic agent other than ifosfamide. ${ }^{13}$ In our cohort, ifosfamide-treated survivors without other potentially nephrotoxic agents association did not present with renal dysfunction.

The majority of patients affected by childhood sarcoma receive one or more potentially nephrotoxic drugs for cancer treatment. Since the 1980s, ifosfamide was introduced in most of the treatment protocols for childhood soft tissue and bone sarcomas. Ifosfamide may cause glomerular and tubular damage during or after administration, but there are few studies that have examined the long-term nephrotoxicity. To examine the long term renal toxicity of ifosfamide, we selected in our cohort of adult sarcoma all cases who had received ifosfamide but no platinum-based drugs (cisplatin or carboplatin) or methotrexate and do not have a diagnosis of genito-urinary rhabdomyosarcoma. No tubular dysfunction, no CKD and no eGFR $<90 \mathrm{~mL} / \mathrm{min} / 1.73 \mathrm{~m}^{2}$ have been detected in this population. This data is not consistent with most data that has been reported in the literature, so far. However, most published studies have reported data on ifosfamide nephrotoxicity after a short term follow-up. Skinner et al conducted a multi-center cross-sectional study evaluating the effects of ifosfamide treatment in 148 cancer survivors at a median time of follow-up of 6 months following the completion of chemotherapy; they found that $50 \%$ of the sample population had GFR $<90 \mathrm{~mL} / \mathrm{min} /$ $1.73 \mathrm{~m}^{2}, 9 \%<60 \mathrm{~mL} / \mathrm{min} / 1.73 \mathrm{~m}^{2}$, and $21 \%$ had hypophosphatemia. ${ }^{14}$ Although the sample size was larger than that in the present study, the follow-up was very short. Rossi et al investigated renal function in 75 patients at a median of follow-up of 31 months, they reported a Fanconi syndrome in $7 \%$ of the cases and a subclinical tubular toxicity in $9 \%$ of the cases. ${ }^{15}$ Loebstein et al screened 174 patients affected by different tumors who had received ifosfamide, that was associated with cisplatin in $70 \%$ 
of the cases; they found renal tubular toxicity in $41.4 \%$ of the cases and glomerular toxicity in $6.3 \%$ of the cases, at short term follow-up. ${ }^{16}$ Stohr et al reported a lower grade of nephrotoxicity. The authors screened 593 patients treated for sarcomas at median follow-up of 19 months and reported a tubular toxicity only in $4.6 \%$ of the cases. ${ }^{17}$ There are two published very long-term follow up studies, that had investigated ifosfamide nephrotoxicity at a longer follow-up. ${ }^{18,19}$ Oberlin et al studied 183 patients after a median follow-up of 10 years. ${ }^{18}$ The authors found a GFR $<90 \mathrm{~mL} / \mathrm{min} / 1.73 \mathrm{~m}^{2}$ in $21 \%$ of the cases, a GFR $<60 \mathrm{~mL} / \mathrm{min} / 1.73 \mathrm{~m}^{2}$ in $0.5 \%$ and a tubular dysfunction in $10.5 \%$. They concluded that despite late common tubular toxicity after ifosfamide treatment, this risk is mild and when present, toxicity is moderate when the cumulative ifosfamide dose is low (91\% of the patients had received $60 \mathrm{~g} / \mathrm{m}^{2}$ or less). ${ }^{18}$ In this study, it was not specified if bladder/prostate RMS survivors were included among the analyzed cases. Oberlin et al also found that the length of follow-up between the end of ifosfamide treatment and investigations was linked with a risk of proximal tubular damage. This feature does not correlate with other studies which described a possible improvement of renal function with time in some children. ${ }^{19,20}$ Skinner et al reported a longitudinal study on 25 patients diagnosed with sarcomas, who were treated with a median cumulative ifosfamide dose of $104 \mathrm{~g} / \mathrm{m}^{2} .{ }^{19}$ They suggested that tubular toxicity improves over a period of several years, although similar recovery does not appear to occur in glomerular function. Indeed, they found that clinically significant tubular toxicity had resolved by 10 years whereas GFR $<60 \mathrm{~mL} / \mathrm{min} / 1.73 \mathrm{~m}^{2}$ was found in $13 \%$ of patients. However, $8 \%$ of these cases underwent high dose chemotherapy or total body irradiation. ${ }^{19}$ In the present study, we were not able to detect an eventual improvement of renal function in our cases, because we did not perform a longitudinal study with the same outcomes. At cross-sectional study, survivors treated with ifosfamide as the only nephrotoxic agent did not present glomerular or tubular toxicity at long-term follow-up. A major limitation of this study was that it was a single institution study with a small number of cases, nevertheless the survivors have received an exhaustive renal function assessment at long-term follow-up.

Hypertension is another important outcome related to renal function. In our study, the prevalence of hypertension was $13.3 \%$ in adult sarcoma survivors. It was higher than that we found in the cohort of adult survivors from unilateral non-syndromic renal tumor, ${ }^{21}$ although this difference was not statistically significant. The possible cause could be related to the higher prevalence of overweight/obesity among adult survivors of sarcoma than that among uninephrectomized cases. ${ }^{20}$ Overweight and obesity are known risk factors for hypertension in the general population. We found a statistically significant correlation between systolic BP and BMI in sarcoma survivors.

Cardoos-Ubbink et al also demonstrated that BMI is the most important risk factor for hypertension following treatment of childhood cancer, emphasizing the need for cancer survivors to maintain a normal weight, although a recent study on a large number of survivors found that BP was substantially higher in all diagnosis groups than expected on the basis of age-, sex-, race/ethnicity-, and BMI-specific population rates. $^{22,23}$

In conclusion, in the present study long-term sarcoma survivors previously treated with ifosfamide as the only nephrotoxic agent do not seem to be at high risk for 
nephrotoxicity. This finding has not been reported so far, it could be conditioned by the small number of cases, and further studies including a larger number of adult long-term survivors are required.

\section{Declaration of interest}

All procedures performed in studies involving human participants were in accordance with the ethical standards of the institutional and/or national research committee and with the 1964 Helsinki declaration and its later amendments or comparable ethical standards. Informed consent was obtained from all individual participants included in the study.

\section{References}

[1] Gatta G, Zigon G, Capocaccia R, et al. EUROCARE Working Group: survival of European children and young adults with cancer diagnosed 1995-2002. Eur J Cancer.2009;45(6): 992-1005.

[2] Geenen MM, Cardous-Ubbink MC, Kremer LC, et al. Medical assessment of adverse health outcomes in long-term survivors of childhood cancer. JAMA. 2007;297(24): $2705-2715$.

[3] Oeffinger KC, Mertens AC, Sklar CA, et al. Childhood Cancer Survivor Study: chronic health conditions in adult survivors of childhood cancer. N Engl J Med. 2006;355(15): $1572-1582$.

[4] Dekkers IA, Blijdorp K, Cransberg K, et al. Long-term nephrotoxicity in adult survivors of childhood cancer. Clin J Am Soc Nephrol. 2013;8(6):922-929.

[5] Skinner R. Late renal toxicity of treatment for childhood malignancy: risk factors, long-term outcomes, and surveillance. Pediatr Nephrol. 2018;33(2):215-225.

[6] Knijnenburg SL, Jaspers MW, van der Pal HJ, et al. Renal dysfunction and elevated blood pressure in long-term childhood cancer survivors. Clin J Am Soc Nephrol. 2012;7(9): $1416-1427$.

[7] Jones DP, Spunt SL, Green D, Springate JE; Children's Oncology Group. Renal late effects in patients treated for cancer in childhood: a report from the Children's Oncology Group. Pediatr Blood Cancer. 2008;51(6):724-731.

[8] Mulder RL, Knijnenburg SL, Geskus RB, et al. Glomerular function time trends in longterm survivors of childhood cancer: a longitudinal study. Cancer Epidemiol Biomarkers Prev. 2013;22(10):1736-1746.

[9] Knijnenburg SL, Mulder RL, Schouten-Van Meeteren AY, et al. Early and late renal adverse effects after potentially nephrotoxic treatment for childhood cancer. Cochrane Database Syst Rev. 2013;8(10):CD008944.

[10] Miller WG, Myers GL, Ashwood ER, et al. Creatinine measurement: state of the art in accuracy and interlaboratory harmonization. Arch Pathol Lab Med. 2005;129:297-304.

[11] Levey AS, Stevens LA, Schmid CH, et al. CKD-EPI (Chronic Kidney Disease Epidemiology Collaboration). A new equation to estimate glomerular filtration rate. Ann Intern Med. 2009;150(9):604-612.

[12] Kidney Disease: Improving Global Outcomes (KDIGO) CKD Work Group. KDIGO 2012 clinical practice guideline for the evaluation and management of chronic kidney disease. Kidney Inter Suppl. 2013;3:1-150.

[13] Raney B, Anderson J, Jenney M, et al. Late effects in 164 patients with rhabdomyosarcoma of the bladder/prostate region: a report from the international workshop. J Urol. 2006;176(5):2190, discussion 2194-2195.

[14] Skinner R, Cotterill SJ, Stevens MC; United Kingdom Children's Cancer Study Group. Risk factors for nephrotoxicity after ifosfamide treatment in children: a UKCCSG Late Effects Group study. Br J Cancer. 2000;82:1636-1645. 
[15] Rossi R, Pleyer J, Schäfers P, et al. Development of ifosfamide-induced nephrotoxicity: prospective follow-up in 75 patients. Med Pediatr Oncol. 1999;32(3):177-182.

[16] Loebstein R, Atanackovic G, Bishai R, et al. Risk factors for long-term outcome of ifosfamide-induced nephrotoxicity in children. J Clin Pharmacol. 1999;39(5):454-461.

[17] Stöhr W, Paulides M, Bielack S, et al. Ifosfamide-induced nephrotoxicity in 593 sarcoma patients: a report from the Late Effects Surveillance System. Pediatr Blood Cancer. 2007;48(4):447-452.

[18] Oberlin O, Fawaz O, Rey A, et al. Long-term evaluation of Ifosfamide-related nephrotoxicity in children. J Clin Oncol. 2009;27(32):5350-5355.

[19] Skinner R, Parry A, Price L, et al. Glomerular toxicity persists 10 years after ifosfamide treatment in childhood and is not predictable by age or dose. Pediatr Blood Cancer. 2010;54(7):983-989.

[20] Van Gool S, Brock P, Wijndaele G, et al. Reversible hypophosphatemic rickets following ifosfamide treatment. Med Pediatr Oncol. 1992;20(3):254-257.

[21] Schiavetti A, Altavista P, De Luca L, et al. Long-term renal function in unilateral non-syndromic renal tumor survivors treated according to International Society of Pediatric Oncology protocols. Pediatr Blood Cancer. 2015;62(9):1637-1644.

[22] Cardous-Ubbink MC, Geenen MM, Schade KJ, et al. Hypertension in long-term survivors of childhood cancer: a nested case-control study. Eur J Cancer. 2010;46(4):782-790.

[23] Gibson TM, Li Z, Green DM, et al. Blood pressure status in adult survivors of childhood cancer: a report from the St. Jude Lifetime Cohort Study. Cancer Epidemiol Biomarkers Prev. 2017;26(12):1705-1713. 